\title{
AZtecICE: A New Dawn in EBSD Data Processing
}

\author{
J Goulden $^{1}$, P Trimby ${ }^{1}, \mathrm{~K}$ Thomsen ${ }^{2}$ and K. Mehnert ${ }^{2}$ \\ 1. Oxford Instruments NanoAnalysis, Halifax Road, High Wycombe, UK. \\ 2. ST Developments ApS, Aarhus, Denmark.
}

The scope of applications where electron backscatter diffraction (EBSD) on SEM is used to understand and characterize complex materials has increased significantly over recent years. EBSD is now routinely used in both research and industrial environments, including the development and characterization of new materials, in essential failure analysis and in process improvement. This increase in popularity is in part driven by improvements to the EBSD system and also by increasingly challenging materials applications.

Advances in both hardware and software have improved the EBSD workflow, facilitating its evolution into a mainstream tool. Improvements include ready optimization of set-up functions, and easy to follow software navigators. In addition, the continued development of automated tools for unattended data collection has enabled the collection of data over large sample areas, in both 2D and 3D, resulting in exceptionally large datasets. Similarly, a step change in the performance of EBSD hardware [1] has enabled some of these changes. The groundbreaking capability of the new CMOS based EBSD detectors, such as Symmetry, has completely replaced the legacy CCD based detectors in terms of speed of data collection and the quality of the data collected.

This increase in popularity of EBSD is also driven by sophisticated application needs. The testing and understanding of new materials and components, especially in heavily regulated industries, has rigorous requirements for full materials characterization. For example, the use of additive manufacturing in rapid prototyping of parts in aerospace applications demands equally rapid microstructural characterization. The need to fully characterize a raw material in a process control environment also requires the fast analysis of statistically significant, often large, sample sizes.

The improvements discussed above encompass the reliability and speed of data acquisition, making EBSD a much more accessible technique for many researchers. However, there is also a need to improve the workflow post data acquisition, i.e. the processing and analysis of EBSD data once collected. A detailed interrogation of EBSD data covers an enormous breadth of microstructural characteristics [2]; these can be correlated with fundamental material properties, such as phase fraction, phase distribution and grain and boundary characteristics. Through further interrogation of these data it is possible to generate a variety of additional information about the sample. For example, interrogating small changes in orientations within an individual grain can be used to infer the plastic strain in a sample, and correlated with geometrically necessary dislocation densities.

Here a new powerful, flexible and easy to use processing package for EBSD data, AZtecICE is introduced. This solution combines the strengths of the existing market-leading AZtec user interface with a wealth of intelligent tools for extracting information from EBSD data.

The software is designed for total flexibility, whatever the workflow: specific analysis modes can be switched on and off in the user interface depending on requirements (Figure 1). The workflow through 
which different processing options are selected is transparent and will suit even the most advanced user. As well as delivering a flexible user interaction, the algorithms built into AZtecICE are designed to process large data sets efficiently, through the application of quaternions. The data are now stored using the Hierarchical Data Format (HDF5) providing possibilities for interaction with open source programs.

AZtecICE is a comprehensive processing package, enabling scientists and researchers to answer key materials questions quickly.

\section{References:}

[1] J Goulden, P Trimby and A Bewick, Microscopy and Microanalysis 24(S1) (2018), p. 1128. [2] T Maitland and S Sitzman in "Scanning Microscopy for Nanotechnology, Techniques and Applications" ed. W Zhou and ZL Wang (Springer) p .41.

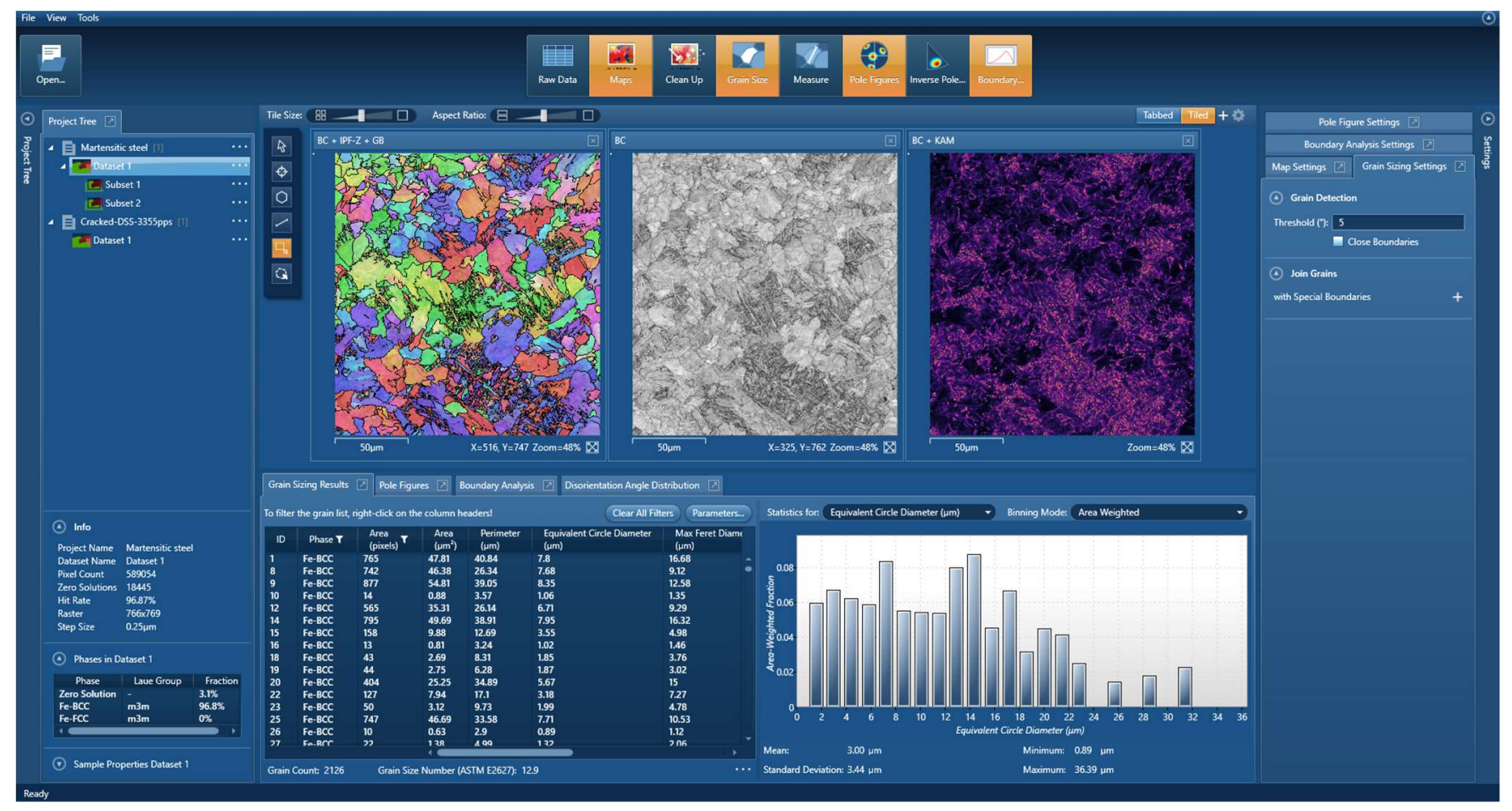

Figure 1. A screenshot showing some of the functions and flexibility of AZtecICE. 